\title{
Geographic variation in prostate cancer survival in New South Wales
}

\section{Xue Qin Yu PhD, MPH Research Fellow \\ Qingwei Luo MAppStats, BSc Data Analyst ${ }^{1}$ \\ David P Smith $\mathrm{PhD}, \mathrm{MPH}$ Associate Professor and Research Fellow \\ Dianne L O'Connell PhD, BMaths(Hons) Professor and Senior \\ Epidemiologist \\ Peter D Baade PhD, AStat Associate Professor and Senior Research Fellow ${ }^{2}$ \\ 1 Cancer Research Division Cancer Council NSW, Sydney, NSW. \\ 2 Viertel Centre for Research in Cancer Control, Cancer Council Queensland \\ Brisbane, QLD. \\ xueqiny@nswcc.org.au}

MJA 2014; 200: 586-590 doi: 10.5694/mjal3.11134
T here have been consistent reports of disparities in cancer survival according to place of residence at diagnosis in Australia. ${ }^{1-5}$ Patients living in rural and remote areas of New South Wales have poorer survival from several major cancers, including prostate cancer, than their city counterparts. ${ }^{6}$ With increasing attention from stakeholders and the media, and with the implementation of health policies and programs designed to reduce urbanrural inequality, ${ }^{7}$ the differential in outcomes from prostate cancer may have reduced.

A recent national study compared prostate cancer incidence, mortality, prostate-specific antigen (PSA) testing and survival for men living within and outside Australian capital cities. ${ }^{1}$ It reported that the survival differential increased over time, so that men living outside the capital cities who were diagnosed with prostate cancer were $24 \%$ more likely to die within 5 years of being diagnosed. However, these results were not adjusted for stage at diagnosis. The same study showed that rates of PSA screening were higher in capital cities. ${ }^{1}$

It has also been reported that rates of advanced prostate cancer are higher in rural NSW, ${ }^{6}$ so the inequality in survival could be explained by higher proportions of men in capital cities being diagnosed with asymptomatic cancers with a low propensity to progress further.

For this reason we expanded the initial NSW study, ${ }^{6}$ which accounted for spread of disease at diagnosis (as an indication of stage), to see whether data for an additional 11 years would show a reduction in urban-rural inequalities in prostate cancer survival. This information will help in assessing whether policies to reduce health inequalities in Australia ${ }^{7}$ have been effective.

Abstract

Objectives: To determine whether the previously reported urban-rural differential in prostate cancer survival remains after adjusting for demographic and clinical factors, and to investigate temporal trends in this differential.

Design, setting and participants: Retrospective population-based survival analysis of 68686 men diagnosed with prostate cancer from January 1982 to December 2007 in New South Wales.

Main outcome measures: Survival rate and relative excess risk (RER) of death over 10 years of follow-up in relation to geographic remoteness after adjusting for other prognostic factors.

Results: Overall, 10-year survival increased during the study period, increasing from $57.5 \%$ in $1992-1996$ and $75.7 \%$ in $1997-2001$ to $83.7 \%$ in $2002-2007$.

The increasing trends were also observed across categories of geographic remoteness and socioeconomic status. Urban-rural differentials were significant $(P<0.001)$ after adjusting for five important prognostic factors, with men living outside major cities having higher risk of death from prostate cancer (RER, 1.18 and 1.32 for inner regional and rural areas, respectively). Socioeconomic status was also a significant factor $(P<0.001)$ for prostate cancer mortality, with the risk of dying being 34\% to $40 \%$ higher for men living in socioeconomically disadvantaged areas than those living in least disadvantaged areas. There was no evidence that this inequality is reducing over time, particularly for men living in inner regional areas.

Conclusions: Despite the increasing awareness of urban-rural differentials in cancer outcomes, little progress has been made. Appropriately detailed data, including details of tumour characteristics, treatment and comorbid conditions, to help understand why these inequalities exist are required urgently so interventions and policy changes can be guided by appropriate evidence.

\section{Methods}

We obtained data from the NSW Central Cancer Registry (CCR) on all first primary prostate cancers that were diagnosed in patients aged $18-84$ years from 1 January 1982 to 31 December 2007 and that were prevalent cases between 1992 and 2007. We excluded cases that were reported through death certificate only or first identified postmortem.

\section{Study variables}

The outcome variable was all-cause survival time after a diagnosis of prostate cancer. Survival status was obtained through record linkage of the cancer cases in the CCR with the death records from the NSW Register of Births, Deaths and Marriages and the National Death Index. All eligible patients were followed up until 31 December 2007, the most recent data available to us.
The primary study variable was place of residence, based on local government area. We categorised place of residence into major cities, inner regional and "rural" (a grouping of outer regional, remote and very remote) areas based on the Australian Standard Geographic Classification Remoteness Structure ${ }^{8}$ (Appendix 1; online at mja.com.au).

We included two additional areabased measures. First, we used agestandardised prostate cancer incidence rates as a surrogate for PSA testing activities, because much of the urban-rural variation in prostate cancer incidence rates may reflect differences in the intensity of PSA screening between geographic areas. ${ }^{9}$ Second, we used the Index of Relative Socioeconomic Disadvantage by local government area (LGA), derived from the 2001 Census, as a measure of arealevel socioeconomic status (SES). ${ }^{10}$ For each LGA, these two measures were 
ranked and then grouped into three levels (high, medium and low), with the cut-points chosen to ensure similar sized populations in each group.

Additional variables included were age at diagnosis, categorised into three groups (18-64 years, 65-74 years and 75-84 years) and spread of disease at diagnosis categorised into three groups (localised, non-localised and unknown).

\section{Ethics approval}

Ethics approval was obtained from the NSW Population and Health Service Research Ethics Committee (reference number: 2009/03/139).

\section{Statistical analysis}

Survival relative to the general population was calculated using period analysis, ${ }^{11}$ with cancer cases under observation in each of three "at-risk" periods - 1992-1996, 1997-2001 and 2002-2007. We estimated expected survival for each at-risk period and degree of geographic remoteness using data on all-cause mortality and numbers of males in the NSW population by age in years, LGA and calendar year provided by Australian Bureau of Statistics. A publicly available set of STATA syntax programs were used for estimating relative survival (http:// www.pauldickman.com/survival/strs. pdf).

To adjust for other prognostic factors, we used a Poisson regression model ${ }^{12}$ to examine geographic variation in relative survival. The relative excess risk (RER) of death derived from this model is the ratio of the excess risk of death in a given region to that of the reference (major cities in this study) after controlling for other factors including age, spread of disease at diagnosis, prostate cancer incidence, at-risk period, year of follow-up (up to 10 years) and SES. A two-sided, loglikelihood ratio test result with a $P$ value $<0.05$ indicated statistical significance. An absolute measure - number of excess deaths ${ }^{5}$ - was calculated as the difference between the number of excess deaths observed and the number expected if all patients with prostate cancer in NSW had the same probability of survival as those in the major cities or the least disadvantaged SES group.

We conducted several further analyses:

\begin{tabular}{|c|c|c|c|c|c|}
\hline \multirow[b]{2}{*}{ Characteristic } & \multirow[b]{2}{*}{ Total } & \multicolumn{3}{|c|}{ Geographic remoteness* } & \multirow[b]{2}{*}{$P^{\dagger}$} \\
\hline & & Major cities & Inner regional & Rural & \\
\hline Number of patients & 68686 & 46545 & 16431 & 5710 & \\
\hline Age at diagnosis & & & & & $<0.001$ \\
\hline$<65$ years & $20227(29.4 \%)$ & $30.1 \%$ & $27.9 \%$ & $28.3 \%$ & \\
\hline $65-74$ years & $29255(42.6 \%)$ & $42.1 \%$ & $43.8 \%$ & $43.2 \%$ & \\
\hline $75-84$ years & $19204(28.0 \%)$ & $27.8 \%$ & $28.3 \%$ & $28.5 \%$ & \\
\hline Spread of disease & & & & & $<0.001$ \\
\hline Localised & 32774 (47.7\%) & $49.5 \%$ & $44.3 \%$ & $43.4 \%$ & \\
\hline Non-localised & $6749(9.8 \%)$ & $9.9 \%$ & $9.4 \%$ & $10.8 \%$ & \\
\hline Unknown & $29163(42.5 \%)$ & $40.7 \%$ & $46.3 \%$ & $45.8 \%$ & \\
\hline Incidence rate $\ddagger$ & & & & & $<0.001$ \\
\hline Low & $15894(23.1 \%)$ & $25.2 \%$ & $18.1 \%$ & $20.7 \%$ & \\
\hline Moderate & $22977(33.5 \%)$ & $36.8 \%$ & $30.8 \%$ & $13.6 \%$ & \\
\hline High & $29815(43.4 \%)$ & $37.9 \%$ & $51.1 \%$ & $65.8 \%$ & \\
\hline Socioeconomic status & & & & & $<0.001$ \\
\hline Least disadvantaged & $23498(34.2 \%)$ & $46.5 \%$ & $10.1 \%$ & $3.0 \%$ & \\
\hline Middle group & $22807(33.2 \%)$ & $28.2 \%$ & $45.6 \%$ & $38.4 \%$ & \\
\hline Most disadvantaged & $22381(32.6 \%)$ & $25.3 \%$ & $44.3 \%$ & $58.6 \%$ & \\
\hline
\end{tabular}

* Based on patients' place of residence at diagnosis using the remoteness classification with the rural category representing outer regional, remote and very remote areas. †Based on $\chi^{2}$ test. $\ddagger$ Directly age-standardised to the 2001 Australian standard population.

- we added an interaction term between the geographic remoteness and at-risk period to the model to allow the effect of geographic remoteness to change over time and then used a likelihood ratio test between the nested models to assess if this interaction was significant;

- we compared two models (one including SES and another without) to examine the effect of SES on the geographic variation in survival over time;

- we performed a sensitivity analysis by assuming all cases with unknown stage had localised cancers, or non-localised cancers to examine the effect of unknown stage on geographic variation in outcomes; and

- we estimated age-standardised prostate cancer mortality rates over 1992-2007 by geographic location to consider the possible impact of lead-time bias on survival introduced by urban-rural differences in the intensity of PSA testing, as lead-time bias does not affect mortality rates.
Analyses were performed using Stata statistical software, version 11.1 (StataCorp).

\section{Results}

There were 68959 men aged 18-84 years diagnosed with prostate cancer from 1 January 1982 to 31 December 2007; 273 cases were excluded because they had been reported to the CCR through death certificate only or identified postmortem. Of the 68686 men included in this study, over twothirds (67.8\%) lived in major cities and about a quarter (23.9\%) in inner regional areas; $47.7 \%$ had localised disease and the stage was unknown in $42.5 \%$. Bivariate analysis showed that all demographic and clinical factors were highly associated with geographic remoteness (Box 1). Patients living in inner regional or rural areas were less likely to have localised disease and more likely to be diagnosed with cancer of an unknown stage than those in major cities. Incidence rates were higher in inner regional and rural areas than in major cities.

The 10-year relative survival rate for men diagnosed with prostate cancer increased during the entire study 
period, from $57.5 \%$ in $1992-1996$ and $75.7 \%$ in $1997-2001$ to $83.7 \%$ in 2002 2007. The increased survival over time was also observed across categories of geographic remoteness and SES (Box 2).

The results of our multivariate analysis show that, before adjusting for other prognostic factors, the RER of death was higher for men living outside major cities (1.36 for inner regional and 1.66 for rural; Appendix 2, online at mja.com.au). Each step of further adjustment for potential prognostic factors, first at-risk period and age group, then disease spread and incidence rates and, finally, SES, progressively reduced the RER of death from 1.36 to 1.18 for inner regional areas and from 1.66 to 1.32 for rural areas (compared with major cities). However, geographic remoteness remained a significant factor $(P<0.001)$. The results in our Model 3 (Appendix 2), which adjusts for the potential prognostic factors above, suggest that these factors explain some of the urban-rural variation in prostate cancer survival but not all.

SES was also a significant prognostic factor, with men living in the middle and most disadvantaged areas having higher mortality risks (RER, 1.34 and 1.40 , respectively) than those living in affluent areas $(P<0.001)$.

Of the total 7291 deaths attributable to prostate cancer, 709 (10\%) could have been avoided if the urban-rural disparity was eliminated, and 1908 (26\%) could have been avoided if the SES disparity was eliminated.

Results over time showed that geographic variation was significant across all three at-risk periods (Box 3 ). In each period, the adjusted RER was significantly higher for patients living in inner regional and rural areas than for those in major cities, with an upward trend for patients in inner regional areas, while the RER in rural areas increased and then decreased slightly. The interaction between geographic remoteness and at-risk period was marginally significant before adjusting for SES $(P=0.09)$, indicating that the urban-rural differential had changed over time. Adjusting for SES made the interaction become nonsignificant $(P=0.13)$, but the patterns of change in RER remained the same.

The sensitivity analyses found that the urban-rural disparities remained

2 Relative survival $(95 \% \mathrm{Cl}$ ) from prostate cancer in New South Wales by geographic remoteness and socioeconomic status for each of the three at-risk periods* in the period analysis, 1992-2007

A: By geographic remoteness
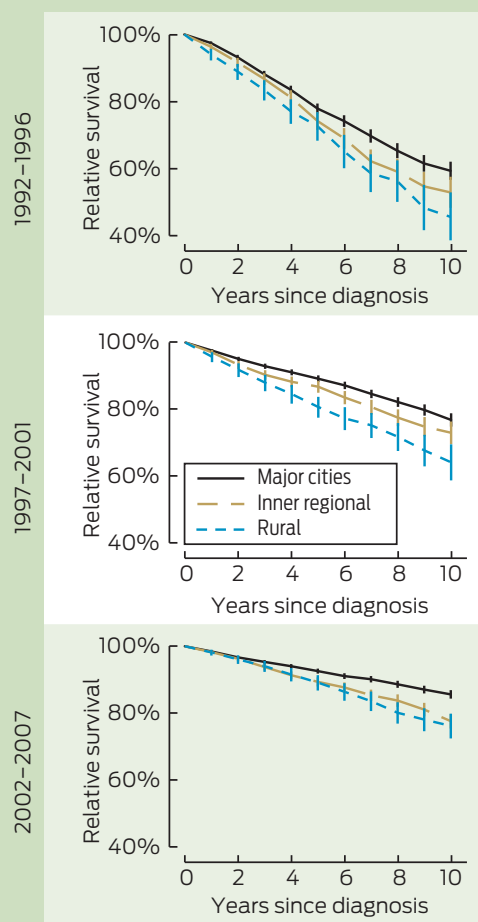

* The "at-risk" periods were when deaths were observed and not necessarily when the cases were diagnosed.

significant for both assumptions $(P<0.001)$ regarding those with unknown spread of disease (data not shown). Mortality rates were consistently lower for men living in major cities over the whole study period 1992-2007 (Appendix 3; online at mja. com.au).

\section{Discussion}

Our findings show that, although the overall survival outlook for men diagnosed with prostate cancer has improved over time, there is still a continuing (and some suggestion of a widening) inequality by place of residence, particularly in inner regional areas compared with major cities. Overall, men living outside major cities of NSW had poorer survival outcomes.

Recent studies have shown that PSA testing is more prevalent in urban areas of Australia. ${ }^{1}$ Thus, urban men may be more likely to be diagnosed with low-risk cancers through PSA testing, which would inflate the observed survival in this group. The large proportion of prostate cancers with unknown spread of disease (43\%) may have camouflaged the true impact of disease spread on the geographic differential. However, the significance of this differential remained unchanged in our sensitivity analyses.

We could not determine a precise reason for the higher incidence of prostate cancer in regional and rural areas of NSW. Geographic patterns of PSA testing in NSW may be different from previously published national patterns. ${ }^{1}$ Also, the increased risk of advanced prostate cancers in regional areas, which are more likely to be detected because of symptoms rather than PSA screening, might override the lower PSA testing rate. While we have tried to account for the impact of PSA testing by adjusting for prostate cancer incidence rates, this may still leave some residual confounding, especially for the small proportion of men $(<5 \%)$ who were diagnosed before PSA testing was introduced on the Medicare Benefits Schedule.

In addition, as was observed nationally, ${ }^{1}$ we found that mortality rates for prostate cancer in NSW were 
3 Relative excess risk of death during the first 10 years after diagnosis with prostate cancer in New South Wales by geographic remoteness, and change over time for each of the three at-risk periods* in the period analysis, 1992-2007

Relative excess risk $(95 \% \mathrm{Cl}) *$

\begin{tabular}{lcc} 
Geographic remoteness & Not adjusted for SES & Adjusted for SES \\
\hline At-risk period 1992-1996 & 1.00 & 1.00 \\
Major cities & $1.23(1.09-1.37)$ & $1.11(0.99-1.25)$ \\
$\quad$ Inner regional & $1.47(1.25-1.72)$ & $1.26(1.07-1.49)$ \\
$\quad$ Rural & 1.00 & 1.00 \\
At-risk period 1997-2001 & $1.29(1.14-1.46)$ & $1.16(1.02-1.32)$ \\
$\quad$ Major cities & $1.72(1.46-2.02)$ & $1.49(1.26-1.76)$ \\
$\quad$ Inner regional & 1.00 & 1.00 \\
$\quad$ Rural & $1.45(1.29-1.63)$ & $1.29(1.14-1.45)$ \\
At-risk period 2002-2007 & $1.41(1.18-1.68)$ & $1.23(1.02-1.47)$ \\
$\quad$ Major cities & & \\
$\quad \begin{array}{l}\text { Inner regional } \\
\text { Rural }\end{array}$ & 0.09 & 0.13 \\
$\begin{array}{l}P \text { value for interaction between } \\
\text { geographic remoteness and } \\
\text { at-risk period }\end{array}$ & & \\
\hline $\begin{array}{l}\text { SES = socioeconomic status. } \\
\text { * Adjusted for age at diagnosis, spread of disease at diagnosis, incidence rate. }\end{array}$ &
\end{tabular}

consistently higher for men living in regional and rural areas (Appendix 3; online at mja.com.au). Therefore, even if the large proportion of prostate cancers with unknown spread of disease conceals a disproportionate amount of over-diagnosis of prostate cancer in urban areas, there remains a mortality inequality that suggests the survival differential was genuine and not simply the result of lead-time bias.

As our results were adjusted for spread of disease at diagnosis, it is unlikely that the urban-rural differential can be explained by different diagnostic practices. This increases the likelihood that there were differences in management practices or other factors that contributed to the survival differences. Our study did not examine treatment, but the evidence for improved survival from any one management strategy in men with localised prostate cancer is equivocal. ${ }^{13}$ Current recommendations for localised disease are based on informed choice, ${ }^{14}$ taking into account factors such as clinical characteristics, life expectancy, quality of life and personal preferences ${ }^{15,16}$ rather than an expectation of increased survival. Differences in access to follow-up services could be one explanation, with several studies suggesting that men with metastatic or recurrent disease who are identified and given salvage treatment earlier

A previous study has shown that men living in rural NSW and diagnosed with prostate cancer between 1993 and 2002 are less likely to have a radical prostatectomy after taking account of age and disease spread; ${ }^{18}$ this is consistent with reports from Western Australia, ${ }^{19}$ Queensland ${ }^{20}$ and nationally. ${ }^{1}$ Disease stage is more consistently recorded in patients who undergo surgery, and this may partly explain why the spread of disease was less likely to be unknown in major cities. ${ }^{21}$

Some of the urban-rural survival differential can be explained by socioeconomic disadvantage, with the size of the urban-rural differential being smaller, but still significantly raised, when area disadvantage was included in the model. This finding is consistent with those of international studies showing that socioeconomically disadvantaged men diagnosed with prostate cancer have poorer survival 22 and higher mortality from prostate cancer. ${ }^{23}$

Despite increasing awareness of and efforts to reduce the urban-rural inequalities in cancer outcomes over the past decade, our study suggests that there is no evidence that urbanrural inequalities in prostate cancer survival have reduced over time. While the lack of data on tumour characteristics, treatment and comorbid conditions have better survival. ${ }^{17}$ make precise interpretation difficult, it remains possible that reduced access to general practitioners and specialist cancer care in regional areas may have contributed to this disparity.

A longer follow-up interval may be needed to observe the impact of recent changes in patterns of prostate cancer diagnosis and management. 24,25 However, there is still a lack of appropriately detailed data to investigate geographic inequalities in the patterns of management of men diagnosed with prostate cancer and their use of health services. In the absence of clear guidelines on optimum management for prostate cancer, these data are required urgently to redress the significant divide in survival outcomes for men diagnosed with prostate cancer in New South Wales.

Competing interests: No relevant disclosures.

Received 02 Sep 2013, accepted 20 Jan 2014.

1 Baade PD, Youlden DR, Coory MD, et al. Urbanrural differences in prostate cancer outcomes in Australia: what has changed? Med J Aust 2011; 194: 293-296.

2 Cramb SM, Mengersen KL, Turrell G, Baade PD. Spatial inequalities in colorectal and breast cancer survival: premature deaths and associated factors. Health Place 2012; 18 : 1412-1421.

3 Dasgupta P, Baade PD, Aitken JF, Turrell G. Multilevel determinants of breast cancer survival: association with geographic remoteness and area-level socioeconomic disadvantage. Breast Cancer Res Treat 2012; 132: 701-710.

4 Yu XQ, O'Connell DL, Gibberd RW, Armstrong BK. A population-based study from New South Wales, Australia 1996-2001: area variation in survival from colorectal cancer. Eur J Cancer 2005; 41: 2715-2721.

5 YU XQ, O'Connell DL, Gibberd RW, Armstrong BK. Assessing the impact of socio-economic status on cancer survival in New South Wales, Australia 1996-2001. Cancer Causes Control 2008; 19: 1383-1390.

6 Jong KE, Smith DP, Yu XQ, et al. Remoteness of residence and survival from cancer in New South Wales. Med J Aust 2004; 180: 618-622.

7 Newman L, Baum F, Harris E. Federal, state and territory government responses to health inequities and the social determinants of health in Australia. Health Promot J Austr 2006; 17: 217-225.

8 Australian Bureau of Statistics. ASGC Remoteness Classification: purpose and use. Canberra: Commonwealth of Australia, 2003.

9 Gregorio DI, Kulldorff M, Sheehan TJ, Samociuk H. Geographic distribution of prostate cancer incidence in the era of PSA testing, Connecticut, 1984 to 1998. Urology 2004; 63: 78-82.

10 Australian Bureau of Statistics. Information paper: An Introduction to Socio-Economic Indexes for Areas (SEIFA), 2006. Canberra: ABS, 2008.

11 Brenner H. Long-term survival rates of cancer patients achieved by the end of the 20th century: a period analysis. Lancet 2002; 360: 1131-1135. 
12 Dickman PW, Sloggett A, Hills M, Hakulinen T. Regression models for relative survival. Stat Med 2004; 23: 51-64.

13 Wilt TJ, MacDonald R, Rutks I, et al. Systematic review: comparative effectiveness and harms of treatments for clinically localized prostate cancer. Ann Intern Med 2008; 148: 435-448.

14 Branney P. White A, Jain S, et al. Choosing health, choosing treatment: patient choice after diagnosis of localized prostate cancer. Urology 2009; 74: 968-971.

15 Cox J, Amling CL. Current decision-making in prostate cancer therapy. Curr Opin Urol 2008; 18 275-278.

16 Singh J, Trabulsi EJ, Gomella LG. The quality-oflife impact of prostate cancer treatments. Curr Urol Rep 2010; 11: 139-146.

17 Trock BJ, Han M, Freedland SJ, et al. Prostate cancer-specific survival following salvage radiotherapy vs observation in men with biochemical recurrence after radical prostatectomy. JAMA 2008; 299: 2760-2769.

18 Hayen A, Smith DP, Patel MI, O'Connell DL. Patterns of surgical care for prostate cancer in NSW, 1993-2002: rural/urban and socioeconomic variation. Aust N Z J Public Health 2008; 32: 417-420.

19 Hall SE, Holman CD, Wisniewski ZS, Semmens J. Prostate cancer: socio-economic, geographical and private-health insurance effects on care and survival. BJU Int 2005; 95: 51-58.

20 Baade PD, Youlden DR, Gardiner RA, et al. Factors associated with treatment received by men diagnosed with prostate cancer in Queensland, Australia. BJU Int 2012; 110: E712-E719.
21 Lu-Yao GL, Greenberg ER. Changes in prostate cancer incidence and treatment in USA. Lancet 1994; 343: 251-254.

22 Chu DI, Freedland SJ. Prostate cancer. Socioeconomic status and disparities in treatment patterns. Nat Rev Urol 2010; 7 480-481.

23 Wan N, Zhan FB, Cai Z. Socioeconomic disparities in prostate cancer mortality and the impact of geographic scale. South Med J 2011; 104: 553-559.

24 Evans SM, Millar JL, Davis ID, et al. Patterns of care for men diagnosed with prostate cancer in Victoria from 2008 to 2011. Med J Aust 2013; 198 : 540-555.

25 Smith DP, Supramaniam R, Marshall VR, Armstrong BK. Prostate cancer and prostatespecific antigen testing in New South Wales. Med J Aust 2008; 189: 315-318. 